\title{
Hemorragia suprarrenal que se manifiesta como hematoma escrotal en el recién nacido. A propósito de un caso
} Adrenal hemorrhage presenting as a scrotal hematoma in the newborn. A case report

Dr. Erbu Yarci ${ }^{a}$ Dra. Sema Arayici ${ }^{a}$,Dra. Fatma Nur Sari ${ }^{a}$, Dr. Fuat Emre Canpolat ${ }^{a}$, Dra. Nurdan Uras ${ }^{a}$ Dr. Ugur Dilmen ${ }^{a, b}$

\section{RESUMEN}

La hemorragia suprarrenal neonatal es poco frecuente. Se presenta en el 0,2\% de los recién nacidos. El 10\% de los casos son bilaterales. Puede estar asociada a un traumatismo al nacimiento, peso alto al nacer o un curso neonatal complicado por hipoxia, asfixia, hipotensión o coagulopatía. El hematoma escrotal es una manifestación extremadamente rara de la hemorragia suprarrenal neonatal. La mayoría de los pacientes presentan tumefacción escrotal con coloración azulada. En los recién nacidos, la tumefacción escrotal, con o sin coloración azulada, puede deberse a diferentes causas. Comunicamos un caso inusual de hemorragia suprarrenal neonatal secundaria a asfixia perinatal, que se manifestó con un hematoma escrotal. El diagnóstico de hemorragia suprarrenal neonatal y hematoma escrotal fue ecográfico; el tratamiento conservador evitó la exploración quirúrgica innecesaria.

Palabras clave: asfixia neonatal, enfermedades de las glándulas suprarrenales, hemorragia, hematocele.

http:/ /dx.doi.org/10.5546/aap.2015.e161

\section{INTRODUCCIÓN}

En los recién nacidos, la tumefacción escrotal con o sin coloración azulada puede deberse a diferentes causas, entre ellas hidrocele, hernia inguinal, peritonitis meconial, orquitis, tumores testiculares, traumatismo o torsión testicular. ${ }^{1}$ La hemorragia suprarrenal como consecuencia de asfixia, traumatismo, septicemia y anomalías de la coagulación durante el período perinatal también puede causar hematoma escrotal. ${ }^{2}$ Se notificaron casos aislados de etiología idiopática. Presentamos un caso inusual de hemorragia suprarrenal neonatal secundaria a asfixia perinatal que se manifestó con un hematoma escrotal.

a. División de Neonatología, Hospital Universitario Maternal Zekai Tahir Burak.

b. Departamento de Pediatría, Facultad de Medicina de Yıldırım Beyazıt University.

Angora, Turquía.

Correspondencia:

Dr. Erbu Yarci: erbuyarci@yahoo.com

Financiamiento: Ninguno.

Conflicto de intereses: Ninguno que declarar.

Recibido: 25-11-2014

Aceptado: 26-1-2015

\section{CASO CLÍNICO}

Recién nacido de sexo masculino de 3400 g; parto vaginal complicado por distocia de hombros; requirió intubación al nacer y tuvo signos de lesión isquémica hipóxica con afectación multisistémica. A los seis días de vida, el escroto derecho se presentaba tumefacto y color azulado (Figura 1). La ecografía de escroto mostró un hematoma en el escroto derecho con testículos normales (Figura 2). La ecografía abdominal reveló la presencia de masas quísticas suprarrenales bilaterales sugestivas de una hemorragia suprarrenal. Los resultados del hemograma fueron los siguientes: hemoglobina $16,2 \mathrm{~g} / \mathrm{dl}$, hematocrito 51\%, leucocitos $25000 /$ $\mathrm{mm}^{3}$ y plaquetas $269000 / \mathrm{mm}^{3}$. El tiempo de protrombina y el tiempo de tromboplastina parcial activado mostraron valores normales. El tratamiento del recién nacido fue conservador y se programó el control mediante exámenes ecográficos regulares. La ecografía de seguimiento

FIGURA 1. Tumefacción y coloración azulada de la ingle y del hemiescroto derechos 
mostró la disminución en el tamaño de la hemorragia suprarrenal con resolución completa. Los resultados de los exámenes no mostraron anomalías en las funciones suprarrenales ni en el eje hipotálamo-suprarrenal.

\section{DISCUSIÓN}

La hemorragia suprarrenal neonatal es poco frecuente; se presenta en el $0,2 \%$ de los recién nacidos y entre el $0,05 \%$ y $0,14 \%$ de las autopsias neonatales. El 10\% de los casos son bilaterales. ${ }^{2}$ En general, se manifiesta en el nacimiento o durante los primeros días de vida. ${ }^{3}$ En el recién nacido, la glándula suprarrenal es muy grande y vulnerable al daño vascular. ${ }^{4}$ Esta patología puede estar asociada a un traumatismo al nacimiento, peso alto al nacer o un curso neonatal complicado por hipoxia, asfixia, hipotensión o coagulopatía. También puede ocurrir de manera espontánea. ${ }^{5}$

Las manifestaciones clínicas de la hemorragia suprarrenal neonatal son variables; dependen fundamentalmente del grado de hemorragia y de la cantidad de corteza suprarrenal afectada por la hemorragia. ${ }^{2,6}$ Entre las manifestaciones más frecuentes se encuentran la anemia, masa abdominal, ictericia y, en menor frecuencia, la tumefacción y el cambio de color en la piel del escroto. ${ }^{5,7} \mathrm{Si}$ el sangrado es moderado, la

FIGURA 2. Ecografía abdominal que muestra la colección de sangre alrededor de las glándulas suprarrenales derecha e izquierda. Ambas se observan agrandadas y heterogéneas, imagen compatible con hemorragia suprarrenal bilateral

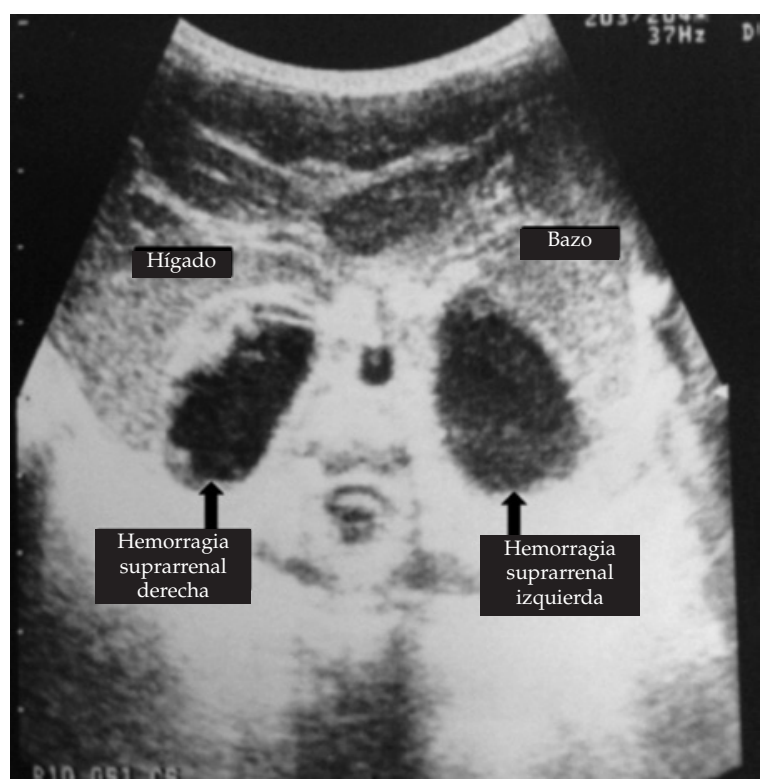

sangre permanece en la cápsula suprarrenal. Una cantidad excesiva de sangre puede provocar la rotura y consecuente invasión de sangre en la cavidad peritoneal o el espacio retroperitoneal. La sangre llega al escroto a lo largo del conducto peritoneo vaginal permeable o por disección del tejido del retroperitoneo. , $^{2,5}$

El hematoma escrotal es una manifestación extremadamente rara de la hemorragia suprarrenal neonatal. La mayoría de los pacientes presentan tumefacción escrotal con coloración azulada. Esta apariencia no es habitual en los recién nacidos y puede deberse a diferentes causas, entre ellas, la presencia de hidrocele, traumatismo testicular con edema testicular, hernia inguinal, tumores congénitos y peritonitis meconial. También se debe tener en cuenta la torsión aguda y subaguda del cordón espermático. ${ }^{9}$ Varios autores recomiendan considerar el cuadro escrotal agudo como torsión testicular para mejorar el salvataje testicular mediante una exploración quirúrgica rápida. ${ }^{10,11}$ Algunos de los casos notificados de hemorragia suprarrenal escrotal y de hematoma escrotal fueron sometidos a cirugías innecesarias. En los recién nacidos, es importante evitar la exploración quirúrgica innecesaria durante el diagnóstico diferencial de tumefacción escrotal y coloración azulada del escroto. ${ }^{12}$

En el caso que describimos, la evaluación ecográfica del escroto mostró un hematoma escrotal derecho con testículos normales; el examen ecográfico con Doppler también fue normal. La evaluación ecográfica abdominal reveló la presencia de masas quísticas suprarrenales bilaterales sugestivas de hemorragia suprarrenal. En cuanto al diagnóstico diferencial, es necesario realizar una evaluación ecográfica de ambos testículos y del abdomen. ${ }^{13-15}$

De los 29 casos de hemorragia suprarrenal neonatal y hematoma escrotal publicados en la bibliografía, 9 fueron intervenidos. En todos los casos abordados con cirugía, la decisión de realizar una intervención quirúrgica se basó solo en el examen físico sugestivo de torsión testicular. ${ }^{14}$

En todos los casos, se encontraron testículos normales y la ecografía abdominal postoperatoria reveló la presencia de una hemorragia suprarrenal ipsilateral. El tratamiento conservador basado en las experiencias previas del médico y la sospecha de hemorragia suprarrenal neonatal como causa de tumefacción escrotal evitó la exploración quirúrgica innecesaria. ${ }^{12}$ 
A modo de conclusión, la hemorragia suprarrenal puede presentarse como tumefacción escrotal imitando un escroto agudo. El diagnóstico diferencial entre esta patología y otras causas de tumefacción escrotal se basa en los antecedentes clínicos y el examen físico y ecográfico de ambos testículos y del abdomen. El tratamiento conservador evita la exploración quirúrgica innecesaria del escroto.

\section{REFERENCIAS}

1. Gonçalves R, Abuabara A, Abuabara RF, Feron CA. Scrotal hematoma as a sign of adrenal hemorrhage in newborns. Sao Paulo Med J 2011;129(2):113-5.

2. Bergami G, Malena S, Di Mario M, Fariello G. Echography in the follow-up of neonatal adrenal hemorrhage. The presentation of 14 cases. Radiol Med 1990;79(5):474-8. Italian.

3. Navarro OM, Daneman A. Congenital and Neonatal Conditions. In: Coley BD, ed. Caffey's pediatric diagnostic imaging. 12 $2^{\text {th }}$ ed. Philadelphia: Elsevier Saunders; 2013. Págs.1274-9.

4. Amoury RA, Barth GW, Hall RT, Rhodes PG, et al. Scrotal ecchymosis: sign of intraperitoneal hemorrhage in the newborn. South Med J 1982;75(12):1471-5.

5. Duman N, Oren H, Gülcan H, Kumral A, et al. Scrotal hematoma due to neonatal adrenal hemorrhage. Pediatrics Int 2004;46(3):360-2.
6. Khuri FJ, Alton DJ, Hardy BE, Cook GT, et al. Adrenal hemorrhage in neonates: report of 5 cases and review of the literature. J Urol 1980;124(5):684-7.

7. Liu KW, Ku KW, Cheung KL, Chan YL. Acute scrotal swelling: a sign of neonatal adrenal haemorrhage. J Paediatr Child Health 1994;30(4):368-9.

8. Miele V, Galluzzo M, Patti G, Mazzoni G, et al. Scrotal hematoma due toneonatal adrenal hemorrhage: the value of ultrasonography in avoiding unnecessary surgery. Pediatric Radiol 1997;27(8):672-4.

9. Adorisio O,Mattei R,Ciardini E,CentonzeN, at al. Neonatal adrenal hemorrhage mimicking an acute scrotum. JPerinatol 2007;27(2):130-2.

10. Das S, Singer A. Controversies of perinatal torsion of the spermatic cord: a review, survey and recommendations. J Urol 1990;143(2):231-3.

11. Al-Salem AH. Intra-uterine testicular torsion: early diagnosis and treatment. BJU Int 1999;83(9):1023-5.

12. Lai LJ, Chen LM, Chu PY, Tseng MH, et al. Neonatal adrenal hemorrhage associated with scrotal hematoma: an unusual case report and literature review. Pediatr Neonatol 2012;53(3):210-2.

13. Ibanez Godoy I, Mora Navarro D, Delgado Rioja MA, Losada Martinez A, et al. Hematoma escrotal unilateral. An Pediatr (Barc) 2004;60(5):477-8.

14. Anding R, Fastnacht-Urban F, Walz PH. 'Acute scrotum' in the neonate. Adrenal haemorrhage as cause. Urologe $A$ 2000;39(1):48-51. German.

15. Giacoia GP, Cravens JD. Neonatal adrenal hemorrhage presenting as scrotal hematoma. J Urol 1990;143(3):567-8. 